\title{
ON SOME STRATEGIES FOR COMPUTER SIMULATION OF THE WAVE PROPAGATION USING FINITE DIFFERENCES I. ONE-DIMENSIONAL FDTD METHOD
}

\author{
Lubomír Šumichrast *
}

Some strategies used in the computer simulation of wave phenomena by means of finite differences in time-domain (FDTD) method are reviewed and discussed here. It is shown that the wave equation in its discretized form possesses different properties in comparison with the true differential formulation. In this part the issues of stability and numerical dispersion are thoroughly investigated for the one-dimensional case represented here by waves on transmission lines and transversal electromagnetic plane wave.

K e y w o r d s: wave phenomena, computer simulation, finite differences in time domain (FDTD) method

\section{INTRODUCTION}

The solution of partial differential equations by numerical methods is subject of many classical mathematical books. The approximation of derivatives by finite differences in differential equations is one of the most transparent and straightforward methods used. The mathematical aspects of the method are in depth treated in eg [1-4]. The special aspects of numerical modeling of the wave propagation are from the engineering point of view treated in many books too, only a few mentioned here from the whole huge palette available up to date are eg [5-8]. The articles in journals can be counted to thousands and the citation of only the most important ones would be longer than this whole paper. The interested reader can, using available search machines, easily find them on himself. However the seminal work of Yee [9] on the explicit FDTD method for vectorial wave components in three dimensions - probably the most cited publication in the field - is certainly worth to be mentioned. In this tutorial paper the substantial features of the FDTD method will be reviewed and thoroughly analysed, first for the one dimensional and subsequently for the more dimensional cases.

\section{WAVE PHENOMENA OF ELECTROMAGNETICS}

The wave propagation is a typical phenomena occurring in electrodynamics, eg voltage and current waves propagating on transmission lines, or electromagnetic waves propagating in space. The main mathematical vehicle used for description of their common features is the wave equation. Only in a very few cases the solutions of the wave equation can be obtained analytically. In prevailing number of situations some numerical method must be used.

The voltages and currents, $u(z, t), i(z, t)$, on homogeneous and lossless transmission lines are described by the telegraphist equations

$$
\begin{aligned}
R_{0} i(z, t)+L_{0} \frac{\partial i(z, t)}{\partial t} & =-\frac{\partial u(z, t)}{\partial z} \\
G_{0} u(z, t)+C_{0} \frac{\partial i(z, t)}{\partial t} & =-\frac{\partial u(z, t)}{\partial z}
\end{aligned}
$$

where $R_{0}, G_{0}, L_{0}$ and $C_{0}$ are distributed resistance, conductance, inductance and capacitance of the line. By separation of variables in (1) and (2) one arrives to the wave equation of the form

$$
\frac{1}{c^{2}} \frac{\partial^{2} f(z, t)}{\partial t^{2}}+\frac{2 \beta}{c} \frac{\partial f(z, t)}{\partial t}+\alpha^{2} f(z, t)-\frac{\partial^{2} f(z, t)}{\partial z^{2}}=0
$$

with $\alpha=\sqrt{R_{0} G_{0}}, \beta=\frac{1}{2}\left(R_{0} \sqrt{C_{0} / L_{0}}+G_{0} \sqrt{L_{0} / C_{0}}\right)$ and $c=1 / \sqrt{L_{0} C_{0}}$, valid for both $u(z, t)$ and $i(z, t)$.

Assuming the solution in form of a steady harmonic wave-amplitude-distribution with the wavenumber $k$, (and wavelength $\lambda=2 \pi / k)$, ie $f(z, t) \approx \phi(t) \exp (-j k z)$ one easily arrives to the solution of type

$$
f(z, t) \approx \exp (-\beta c t) \exp \{-j(k z \pm \omega t)\}
$$

where: $\quad \omega=c \sqrt{k^{2}+\alpha^{2}-\beta^{2}}$,

representing thus the direct and reverse wave attenuated in time and propagating along $z$ with the phase velocity

$$
v_{p}(k)=\omega / k=c \sqrt{1+\left(\alpha^{2}-\beta^{2}\right) / k^{2}} .
$$

Substitution $f(z, t) \approx f_{0}(z, t) \exp (-\beta c t)$ into (3) leads to the equation

$$
\frac{1}{c^{2}} \frac{\partial^{2} f_{0}(z, t)}{\partial t^{2}}+\left(\alpha^{2}-\beta^{2}\right) f_{0}(z, t)-\frac{\partial^{2} f_{0}(z, t)}{\partial z^{2}}=0
$$

for the non-attenuated part of $f(z, t)$ in form $f_{0}(z, t) \approx$ $\exp \{-j(k z \pm \omega t)\}$.

\footnotetext{
* Slovak University of Technology, Institute of Electrical Engineering, Ilkovičova 3, SK-81219 Bratislava, lubomir.sumichrast@stuba.sk
} 
From (5) the frequency dependence (dispersion) of the wavenumber is

$$
k(\omega)=\sqrt{(\omega / c)^{2}+\beta^{2}-\alpha^{2}},
$$

leading to the phase and group velocity

$$
\begin{gathered}
v_{p}(\omega)=\omega / k=c / \sqrt{1+c^{2} \omega^{-2}\left(\beta^{2}-\alpha^{2}\right)}, \\
v_{g}(\omega)=\mathrm{d} \omega / \mathrm{d} k=c \sqrt{1+c^{2} \omega^{-2}\left(\beta^{2}-\alpha^{2}\right)},
\end{gathered}
$$

with $v_{p} v_{g}=c^{2}$.

For $\beta^{2}-\alpha^{2}>0$, ie for the group velocity higher than the phase velocity, $v_{g}>v_{p}$, we have the case of the anomalous dispersion, while for $\beta^{2}-\alpha^{2}<0$, ie for the group velocity lower than the phase velocity, $v_{g}<v_{p}$, we have the case of the normal dispersion. Since for the transmission line

$$
\beta^{2}-\alpha^{2}=\frac{1}{4}\left(R_{0} \sqrt{C_{0} / L_{0}}-G_{0} \sqrt{L_{0} / C_{0}}\right)^{2} \geq 0
$$

holds, the transmission line with losses is an anomalousdispersion-system (provided the distributed parameters of the line are constants independent of $\omega$ ).

A special case - the dispersion-free line with losses occurs if $\beta^{2}-\alpha^{2}=0$, ie if the condition $R_{0} / G_{0}=L_{0} / C_{0}$ is met. Then $k(\omega)=\omega / c$ and $v_{p}=v_{g}=c$.

In fact, due to the causality theorem, physically dispersion goes always with losses and vice versa. The above mentioned case of dispersion-free line with losses must be understood as an approximation for negligible dispersion only. In an opposite case, when losses are negligible, we may omit the term $\exp (-\beta c t)$ in (4) completely and consider only the dispersion effects as described by (7).

\section{LOSSLESS AND DISPERSION-FREE WAVE PROPAGATION}

For the lossless and dispersion-free line, ie if $R_{0}=0$ and $G_{0}=0$, the telegraphist equations (1) and (2) take the simple form

$$
\begin{aligned}
& L_{0} \frac{\partial i(z, t)}{\partial t}=-\frac{\partial u(z, t)}{\partial z} \\
& C_{0} \frac{\partial u(z, t)}{\partial t}=-\frac{\partial i(z, t)}{\partial z} .
\end{aligned}
$$

The one-dimensional variant of the plane electromagnetic field describing the transversal components of electric and magnetic field vectors $E_{x}, H_{y}$ propagating along the $z$-axis in homogeneous, isotropic and lossless media with permittivity $\varepsilon$ and permeability $\mu$ is described by the Maxwell equations

$$
\begin{aligned}
\mu \frac{\partial H_{y}(z, t)}{\partial t} & =-\frac{\partial E_{x}(z, t)}{\partial z} \\
\varepsilon \frac{\partial E_{x}(z, t)}{\partial t} & =-\frac{\partial H_{y}(z, t)}{\partial z},
\end{aligned}
$$

that are analogous to (12).

Having introduced into (12) the normalised quantities $f(z, t)=u(z, t), g(x, t)=Z_{0} i(x, t)$, with $Z_{0}=\sqrt{L_{0} / C_{0}}$, or into (13) $f(z, t)=E_{x}(z, t), g(z, t)=Z_{0} H_{y}(z, t)$, with $Z_{0}=\sqrt{\mu / \varepsilon}$, both (12) and (13) are represented by the coupled pair of equations of the first order

$$
\frac{\partial f(z, t)}{c \partial t}=-\frac{\partial g(z, t)}{\partial z}, \frac{\partial g(z, t)}{c \partial t}=-\frac{\partial f(z, t)}{\partial z},
$$

where $c=\left(L_{0} C_{0}\right)^{-1 / 2}$, or $c=(\mu \varepsilon)^{-1 / 2}$. From (12), (13), or $(14)$, the one-dimensional wave equation of the type

$$
\frac{1}{c^{2}} \frac{\partial^{2} f(z, t)}{\partial t^{2}}-\frac{\partial^{2} f(z, t)}{\partial z^{2}}=0
$$

can be easily obtained, valid in this form also for $g(z, t)$, $u(z, t), i(z, t), E_{x}(z, t)$, or $H_{y}(z, t)$.

The wave character of solutions of (15) is at best manifested by the impulse response $h(z, t)$ being the solution of

$$
\frac{1}{c^{2}} \frac{\partial^{2} h(z, t)}{\partial t^{2}}-\frac{\partial^{2} h(z, t)}{\partial z^{2}}=\delta(z) \delta(t)
$$

where $\delta(t)$ and $\delta(z)$ are the Dirac delta-impulses in time and space. It can be easily obtained for a case of the infinite homogeneous space in form of the causal time response

$$
h(z, t)=\frac{c}{2} \mathbf{I}(t)\{\mathbf{I}(z+c t)-\mathbf{I}(z-c t)\} .
$$

where $\mathbf{I}(\cdot)$ denotes the unit-step (Heaviside) function. In accordance with the causality principle the impulse response is non-zero only for $t>0$ as represented by $\mathbf{I}(t)$. Both, the leading and trailing edge of the rectangular impulse $\mathbf{I}(z+c t)-\mathbf{l}(z-c t)$ are running away from the origin, representing thus the direct and the reverse wave.

\section{SIGNAL DISTORTION DUE TO DISPERSION EFFECTS}

If the temporal Fourier transform $F(z, \omega)$ of the signal $f(z, t)$ is defined as

$$
F(z, \omega)=\int_{-\infty}^{\infty} f(z, t) \exp (-j \omega t) \mathrm{d} t,
$$

then, in the lossless case, the wave equation (7) for the spectral density $F(z, \omega)$ takes the form

$$
\frac{\partial^{2} F(z, \omega)}{\partial z^{2}}+k^{2}(\omega) F(z, \omega)=0,
$$

where $k(\omega)=\omega / v_{p}$ is the frequency-dependent wavenumber. The solution of (19) for the direct wave reads

$$
F(z, \omega)=F(0, \omega) \exp (-j k z)
$$

where $F(0, \omega)$ is the initial value corresponding to the "input" signal $f(0, t), f(0, t) \Leftrightarrow F(0, \omega)$. The "output" 
signal $f(z, t)$ in time domain is obtained through the inverse Fourier transform, ie

$$
f(z, t)=\frac{1}{2 \pi} \int_{-\infty}^{\infty} F(0, \omega) \exp (-j k z) \exp (j \omega t) \mathrm{d} \omega .
$$

Let us take the "input" signal $f(0, t) \Leftrightarrow F(0, \omega)$ in form of a modulated impulse

$$
f(0, t)=s_{0}(t) \exp \left(j \omega_{0} t\right),
$$

with the modulating complex envelope signal $s_{0}(t)$ and harmonic carrier oscillations $\exp \left(j \omega_{0} t\right)$. The spectral density $F(0, \omega)$ is given as

$$
F(0, \omega)=S_{0}\left(\omega-\omega_{0}\right)
$$

where $S_{0}(\omega) \Leftrightarrow s_{0}(t)$ is the spectral density of $s_{0}(t)$.

The "output" signal given by (21) equals

$$
f(z, t)=\frac{1}{2 \pi} \int_{-\infty}^{\infty} S_{0}\left(\omega-\omega_{0}\right) \exp (-j k z) \exp (j \omega t) \mathrm{d} \omega .
$$

Expanding $k(\omega)$ in case of a dispersive medium into the Taylor series

$$
k(\omega)=k_{0}+\tau_{g}\left(\omega-\omega_{0}\right)+\sum_{n=2}^{\infty} d_{n}\left(\omega-\omega_{0}\right)^{n},
$$

where $k_{0}=k\left(\omega_{0}\right), \tau_{g}=k^{\prime}\left(\omega_{0}\right), d_{n}=k^{(n)}\left(\omega_{0}\right) / n$ !, yields the expression

$$
f(z, t)=s\left(t-z / v_{g}\right) \exp \left[j \omega_{0}\left(t-z / v_{p}\right)\right],
$$

where $v_{p}=\omega_{0} / k_{0}$, and $v_{g}=1 / \tau_{g}$, and

$$
\begin{aligned}
s(t)=\frac{1}{2 \pi} \int_{-\infty}^{\infty} S_{0}(\omega) \exp \left\{-j z\left(d_{2} \omega^{2}\right.\right. & \left.\left.+d_{3} \omega^{3}+\ldots\right)\right\} \\
& \times \exp (j \omega t) \mathrm{d} \omega .
\end{aligned}
$$

The result accordingly (26) means that the carrier wave propagates with the phase velocity $v_{p}$, the modulationenvelope signal $s(t)$ propagates with the group velocity $v_{g}$ and is equal to $s_{0}(t)$ deteriorated by the dispersion terms as given by $(27)$. Thus any other dependance of $k(\omega)$ on $\omega$ than linear, $k \sim \omega$, leads to the deterioration of the "output" signal due to dispersion.

Restricting ourselves only to to the first term of the series in (27) one arrives to the deterioration of the signal form due to dispersion of the second order

$$
s(t)=\frac{1}{2 \pi} \int_{-\infty}^{\infty} S_{0}(\omega) \exp \left(-j d_{2} z \omega^{2}\right) \exp (j \omega t) \mathrm{d} \omega
$$

as indicated by the quadratic phase factor $\exp \left(-j d_{2} z \omega^{2}\right)$.

For the input envelope in a special form of the Gaussian impulse

$$
s_{0}(t)=A_{0} \exp \left(-t^{2} / 4 w_{0}^{2}\right)
$$

with the effective impulse-half-width $w_{0}$, using the Fourier transform identity

$$
\exp \left(-t^{2} / 4 w_{0}^{2}\right) \Leftrightarrow 2 w_{0} \sqrt{\pi} \exp \left(-\omega^{2} w_{0}^{2}\right)
$$

one obtaines

$$
\begin{aligned}
& S(\omega)=S_{0}(\omega) \exp \left(-j d_{2} z \omega^{2}\right)= \\
& 2 w_{0} A_{0} \sqrt{\pi} \exp \left\{-\omega^{2}\left(w_{0}^{2}+j d_{2} z\right)\right\}
\end{aligned}
$$

and its inverse

$$
s(t)=\frac{w_{0} A_{0}}{\sqrt{w_{0}^{2}+j d_{2} z}} \exp \left\{-t^{2} / 4\left(w_{0}^{2}+j d_{2} z\right)\right\} .
$$

It can be further expressed as

$$
s(t)=A \exp \left\{-t^{2} / 4 w^{2}\right\} \exp \left\{j \Omega t^{2}\right\},
$$

where all, $A, w$, and $\Omega$, are functions of $z$, depending on the dimensionless parameter $\Phi=d_{2} z / w_{0}^{2}$

$$
\begin{aligned}
& A=A(z)=A_{0} / \sqrt{1+j \Phi}, \\
& w=w(z)=w_{0} \sqrt{1+\Phi^{2}}, \\
& \Omega=\Omega(z)=\Phi / 4 w^{2}
\end{aligned}
$$

It can be easily seen, that the Gaussian form of the impulse envelope remains preserved, and that due to the dispersion the halfwidth of the impulse increases (impulse is broadened), and its instantaneous carrier frequency $\omega_{\text {inst }}=\omega_{0}+\Omega t$ is linearly swept either to the higher or to the lower frequencies (chirping), depending on the sign of the second order dispersion coefficient $d_{2}$. For $e g$ the case of the transmission line analysed above

$$
k^{\prime \prime}(\omega)=c\left(\beta^{2}-\alpha^{2}\right) /\left[(\omega)^{2}+c^{2}\left(\beta^{2}-\alpha^{2}\right)\right]^{3 / 2}
$$

holds. Speaking optically, for the anomalous dispersion $d_{2}>0$ the so called "blue shift" (towards higher frequencies) in carrier takes place, while for the normal dispersion $d_{2}<0$ the "red shift" (towards lower frequencies) occurs.

\section{EXPLICIT AND IMPLICIT DISCRETE MODELING OF THE WAVE PROPAGATION}

To solve (14), or (15) numerically, first the discretization of the continuous values $f(z, t), g(z, t)$ must be performed, ie one has to deal with the set of discrete values $\left.f\right|_{m} ^{n}=f\left(m \Delta_{z}, n \Delta_{t}\right),\left.g\right|_{m} ^{n}=g\left(m \Delta_{z}, n \Delta_{t}\right)$, on the equidistant grid of the discrete points $z_{m}=m \Delta_{z}$, $t_{n}=n \Delta_{t}$.

The so-called staggered grids are often used when dealing with (14). The discrete values of $f(z, t)$ are taken only for even steps, $\left.f\right|_{2 m} ^{2 n}=f\left(2 m \Delta_{z}, 2 n \Delta_{t}\right)$, and of $g(z, t)$ only for odd steps, $\left.g\right|_{2 m+1} ^{2 n+1}=g\left([2 m+1] \Delta_{z},[2 n+1] \Delta_{t}\right)$. Denoting subsequently $2 \Delta_{z}$ and $2 \Delta_{t}$ as the new discretization intervals one has to deal with two, mutually 
half-step-shifted grids, eg $\left.f\right|_{m} ^{n}=f\left(m \Delta_{z}, n \Delta_{t}\right)$ in the discrete points $z_{m}=m \Delta_{z}, t_{n}=n \Delta_{t}$ and $\left.g\right|_{m+1 / 2} ^{n+1 / 2}=$ $g\left(\left[m+\frac{1}{2}\right] \Delta_{z},\left[n+\frac{1}{2}\right] \Delta_{t}\right)$ in the points $z_{m+1 / 2}=[m+$ $\left.\frac{1}{2}\right] \Delta_{z}, t_{n+1 / 2}=\left[n+\frac{1}{2}\right] \Delta_{t}$. Sometimes instead of doublestaggered grids (in spatial and time direction) a singlestaggered grid in the spatial direction only is used, ie $\left.f\right|_{m} ^{n}$ and $\left.g\right|_{m+1 / 2} ^{n}$ as illustrated in Fig. 1.

(a)

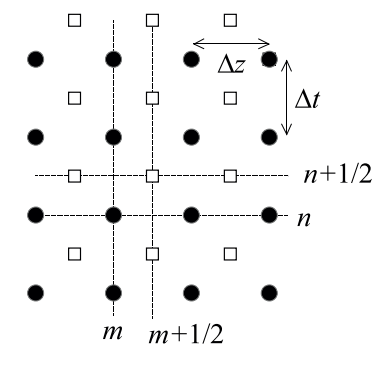

(b)
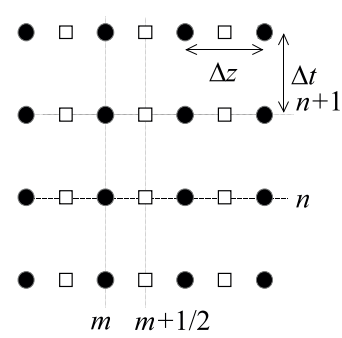

Fig. 1. (a) - double staggered grid, $\bullet=\left.f\right|_{m} ^{n}, \square=\left.g\right|_{m+1 / 2} ^{n+1 / 2}$,

(b) - single staggered grids, $\bullet=\left.f\right|_{m} ^{n}, \square=\left.g\right|_{m+1 / 2} ^{n}$

The numerical algorithm representing (14) is quite simple. The use of the first central differences yields

$$
\begin{aligned}
& \frac{\left.f\right|_{m} ^{n+1}-\left.f\right|_{m} ^{n}}{c \Delta_{t}}=-\frac{\left.g\right|_{m+1 / 2} ^{n+1 / 2}-\left.g\right|_{m-1 / 2} ^{n+1 / 2}}{\Delta_{z}} \\
& \frac{\left.g\right|_{m+1 / 2} ^{n+1 / 2}-\left.g\right|_{m+1 / 2} ^{n-1 / 2}}{c \Delta_{t}}=-\frac{\left.f\right|_{m+1} ^{n}-\left.f\right|_{m} ^{n}}{\Delta_{z}} .
\end{aligned}
$$

Eliminating $\left.g\right|_{m \pm 1 / 2} ^{n \pm 1 / 2}$ from (37) and (38) one arrives to the discrete version of the wave equation (15)

$$
\frac{\left.f\right|_{m} ^{n+1}-\left.2 f\right|_{m} ^{n}+\left.f\right|_{m} ^{n-1}}{c^{2} \Delta_{t}^{2}}-\frac{\left.f\right|_{m+1} ^{n}-\left.2 f\right|_{m} ^{n}+\left.f\right|_{m-1} ^{n}}{\Delta_{z}^{2}}=0 .
$$

An equation of the same form can be obtained for $\left.g\right|_{m \pm 1 / 2} ^{n \pm 1 / 2}$ too, by eliminating $\left.f\right|_{m} ^{n}$ instead of $\left.g\right|_{m \pm 1 / 2} ^{n \pm 1 / 2}$ from (37) and (38).

For the wave propagation simulation either (39) alone, or (37) together with (38) is used. The computations are performed in the two-dimensional computational window. The spatial dimension is for $x \in\left(0, x_{\max }\right)$, with finite number of values $m=0,1,2, \ldots, M$, and the equidistant discretization interval $\Delta_{x}=x_{\max } / M$. The second dimension (the time axis) represents the propagation of the wave in time by the "forward marching" algorithm.

In order to use (39) for the "running time" index $n=$ $0,1,2, \ldots$, the starting initial values $\left.f\right|_{m} ^{0},\left.f\right|_{m} ^{1}$ in all points $m=0,1,2, \ldots, M$ together with the "boundary" values $\left.f\right|_{m} ^{n}$ for $m=0$ and $m=M$ in all time points $n=0,1,2, \ldots$ must be given as sketched in Fig. 2a.

The discrete initial values $\left.f\right|_{m} ^{0},\left.f\right|_{m} ^{1}$ approximate in discrete form the continuous initial conditions $\left.f(x, t)\right|_{t=0}$ and $\partial f(x, t) /\left.\partial t\right|_{t=0}$ approximating $\left.f(x, t)\right|_{t=0}$ by $\left.f\right|_{m} ^{0}$ and $\partial f(x, t) /\left.\partial t\right|_{t=0}$ by $\left(\left.f\right|_{m} ^{1}-\left.f\right|_{m} ^{0}\right) / \Delta_{t}$. The discrete boundary conditions $\left.f\right|_{0} ^{n},\left.f\right|_{M} ^{n}$ approximating $\left.f(x, t)\right|_{x=0}$ by and $\left.f(x, t)\right|_{x=x_{\max }}$ all needed for the unique solution of (15).

When using doubly staggered grids with (37) and (38) the initial time derivative $\partial f(x, t) /\left.\partial t\right|_{t=0}$ is encoded in $\left.g\right|_{m+1 / 2} ^{1 / 2}$, since $\left(\left.f\right|_{m} ^{1}-\left.f\right|_{m} ^{0}\right) / \Delta_{t}=-\left(\left.g\right|_{m+1 / 2} ^{1 / 2}-\right.$ $\left.\left.g\right|_{m-1 / 2} ^{1 / 2}\right) / \Delta_{z}$, as depicted in Fig. 2 b.

For singly staggered grids (Fig. 2c) instead of forward time differences one has to deal with the central differences, since $\left(\left.f\right|_{m} ^{1 / 2}-\left.f\right|_{m} ^{-1 / 2}\right) / \Delta_{t}=-\left(\left.g\right|_{m+1 / 2} ^{0}-\right.$ $\left.\left.g\right|_{m-1 / 2} ^{0}\right) / \Delta_{z}$ form

Equations (37), (38) and (39) can be written in the

$$
\begin{gathered}
\left.\quad f\right|_{m} ^{n+1}=\left.f\right|_{m} ^{n}-b\left(\left.g\right|_{m+1 / 2} ^{n+1 / 2}-\left.g\right|_{m-1 / 2} ^{n+1 / 2}\right) \\
\left.g\right|_{m+1 / 2} ^{n+1 / 2}=\left.g\right|_{m+1 / 2} ^{n-1 / 2}-b\left(f_{m+1}^{n}-\left.f\right|_{m} ^{n}\right) \\
\left.f\right|_{m} ^{n+1}=\left.f\right|_{m} ^{n-1}-\left.2 f\right|_{m} ^{n}+b^{2}\left(\left.f\right|_{m+1} ^{n}-\left.2 f\right|_{m} ^{n}+\left.f\right|_{m-1} ^{n}\right) \\
\text { where } \quad b=c \Delta_{t} / \Delta_{z}
\end{gathered}
$$



(a)

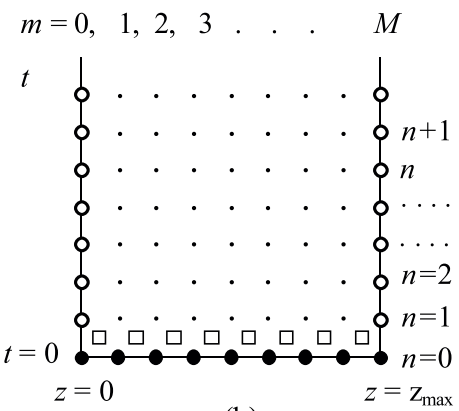

(b)

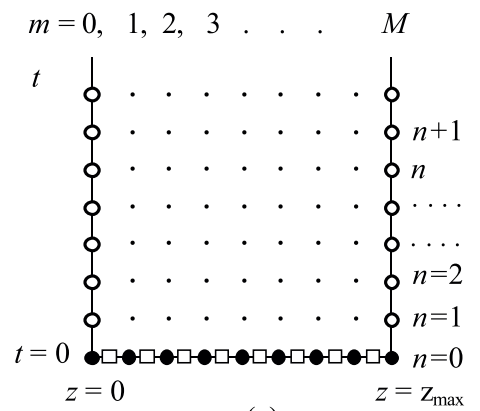

(c)

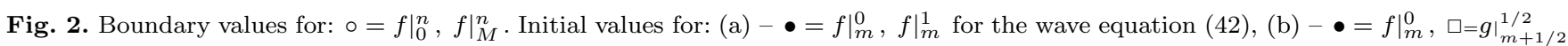
for double-staggered grid accordingly (40) and (41), (c)- • $=\left.f\right|_{m} ^{0}, \square=\left.g\right|_{m+1 / 2} ^{0}$ for single-staggered grid accordingly (44) and (45). 
(a)

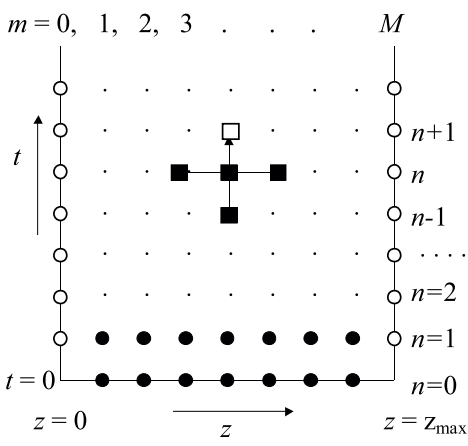

(b)



Fig. 3. Calculations: - - previously calculated values, (a) - explicit scheme $\square-$ the value to be directly calculated, (b) - implicit scheme

- previously calculated values, $\square$ - the values to be resolved

is the so called Courant number. Here the "explicit" nature of the formulation is clearly visible. For the "running index" $n=0,1,2, \ldots$, the new values $\left.f\right|_{m} ^{n+1}$ in the time layer $t_{n+1}=(n+1) \Delta_{t}$ can be explicitly obtained from the known "previous" values $\left.f\right|_{m} ^{n},\left.f\right|_{m} ^{n-1},\left.f\right|_{m-1} ^{n},\left.f\right|_{m+1} ^{n}$, as sketched in Fig. 3a without solving any equations (ie there is no matrix inversion necessity).

When using (42), after having obtained all $\left.f\right|_{m} ^{n}$ values, the values $\left.g\right|_{m+1 / 2} ^{n+1 / 2}$ can be obtained from (40) and (41) provided $\left.g\right|_{1 / 2} ^{1 / 2}$ is given (fixing thus the constant level of all $\left.\left.g\right|_{m+1 / 2} ^{n+1 / 2}\right)$.

One can proceed with the computation also directly using both (40) and (41) intermittently. In the course of computation it yields both the full-step values $\left.f\right|_{m} ^{n}$ and the half-step values $\left.g\right|_{m+1 / 2} ^{n+1 / 2}$ simultaneously. The initial conditions must be given in the form $\left.f\right|_{m} ^{0}$ in all points $m=0,1,2, \ldots, M$ and $\left.g\right|_{m+1 / 2} ^{1 / 2}$ in points $m=$ $0,1,2, \ldots, M-1$ as shown in Fig. $2 \mathrm{~b}$. The boundary conditions are the same as above.

Instead of the explicit formulation the implicit CrankNicolson discretization [10] of (14) can be used. Using the single staggered grid as in Fig. 1b it takes the form

$$
\begin{gathered}
\frac{\left.f\right|_{m} ^{n+1}-\left.f\right|_{m} ^{n}}{c \Delta_{t}}=-\frac{\left.g\right|_{m+1 / 2} ^{n+1}-\left.g\right|_{m-1 / 2} ^{n+1}}{2 \Delta_{z}}-\frac{\left.g\right|_{m+1 / 2} ^{n}-\left.g\right|_{m-1 / 2} ^{n}}{2 \Delta_{z}}, \\
\frac{\left.g\right|_{m+1 / 2} ^{n+1}-\left.g\right|_{m+1 / 2} ^{n}}{c \Delta_{t}}=-\frac{f_{m+1}^{n+1}-\left.f\right|_{m} ^{n+1}}{2 \Delta_{z}}-\frac{f_{m+1}^{n}-\left.f\right|_{m} ^{n}}{2 \Delta_{z}}
\end{gathered}
$$

These two equations can be recast in the following form

$$
\left.f\right|_{m+1} ^{n+1}-\left.\left(2+4 b^{-2}\right) f\right|_{m} ^{n+1}+\left.f\right|_{m-1} ^{n+1}=\left.A\right|_{m} ^{n}
$$

where $\left.A\right|_{m} ^{n}$ is either

$$
\begin{aligned}
& \left.A\right|_{m} ^{n}=8 b^{-1}\left(\left.g\right|_{m+1 / 2} ^{n}-\left.g\right|_{m-1 / 2} ^{n}\right)- \\
& \left.\quad f\right|_{m+1} ^{n}+\left.\left(2-4 b^{-2}\right) f\right|_{m} ^{n}-\left.f\right|_{m-1} ^{n},
\end{aligned}
$$

or, after having eliminated $\left.g\right|_{m \pm 1 / 2} ^{n}$ from (44) and (45),

$$
\begin{array}{r}
\left.A\right|_{m} ^{n}=-2\left[\left.f\right|_{m+1} ^{n}-\left.\left(2-4 b^{-2}\right) f\right|_{m} ^{n}+\left.f\right|_{m-1} ^{n}\right]- \\
\left.f\right|_{m+1} ^{n-1}+\left.\left(2+4 b^{-2}\right) f\right|_{m} ^{n-1}-\left.f\right|_{m-1} ^{n-1},
\end{array}
$$

as it is schematically sketched in Fig. $3 \mathrm{~b}$.

In (48) only the values $\left.f\right|^{n}$ and $\left.f\right|^{n-1}$ in two previous time layers occur. The initial values pertaining to (47) are as in Fig. 2c, while for (48) the initial values as in Fig. 2a must be used.

The set of $M-1$ equations for $m=1,2, \ldots, M-$ 1 defined by (46), with unknowns $\left.f\right|_{m+1} ^{n+1},\left.f\right|_{m} ^{n+1}$ and $\left.f\right|_{m-1} ^{n+1}$, with $\left.f\right|_{0} ^{n+1},\left.f\right|_{M} ^{n+1}$ being the known boundary values, represents a system with the tridiagonal matrix and, denoting $\eta=-\left(2+4 b^{-2}\right)$, reads as follows

$$
\left[\begin{array}{rrrr}
\eta & 1 & & \\
& 1 & \eta & 1 \\
\cdots & \ldots & \ldots \\
& 1 & \eta & 1 \\
& & 1 & \eta
\end{array}\right]\left[\begin{array}{c}
f_{1} \\
f_{2} \\
\ldots \\
f_{M-2} \\
f_{M-1}
\end{array}\right]^{n+1}=\left[\begin{array}{c}
A_{1} \\
A_{2} \\
\ldots \\
A_{M-2} \\
A_{M-1}
\end{array}\right]^{n}-\left[\begin{array}{c}
f_{0} \\
0 \\
\ldots \\
0 \\
f_{M}
\end{array}\right]^{n+1}
$$

This set of equations can be quickly and efficiently solved by the Gauss elimination method (Thomas algorithm) [4] for each subsequent step $n+1$. The inverse matrix pertaining to the tridiagonal matrix is a full matrix. Therefore, even if the matrix in (49) is for each step $n+1$ the same, it is more efficient to perform the Gauss elimination for each step, rather than to invert the matrix once, and for all the subsequent steps multiplying the inverted matrix with the RHS of (49).

After having obtained all $\left.f\right|_{m} ^{n+1}$ the values $\left.g\right|_{m+1 / 2} ^{n+1}$ are subsequently calculated in the explicit way from (45)

$$
\left.g\right|_{m+1 / 2} ^{n+1}=\left.g\right|_{m+1 / 2} ^{n}-\frac{b}{2}\left(\left.f\right|_{m+1} ^{n+1}-\left.f\right|_{m} ^{n+1}+\left.f\right|_{m+1} ^{n}-\left.f\right|_{m} ^{n}\right)
$$

When using $\left.A\right|_{\ldots} ^{n}$ from (47) the values $\left.g\right|_{\ldots} ^{n}$ must be calculated immediately after each $n$-th step has been done, since the values $\left.g\right|_{m+1 / 2} ^{n}$ are needed in (47) for the next $n+1$ step. When proceeding with (48) all values $\left.g\right|_{m+1 / 2} ^{n}$ can be again calculated when all $\left.f\right|_{m} ^{n}$ are known.

\section{POWER CONSERVATION AND NUMERICAL DISPERSION}

The fundamental solution of (15) in form of a complex harmonic wave with angular frequency $\omega$, propagating in 


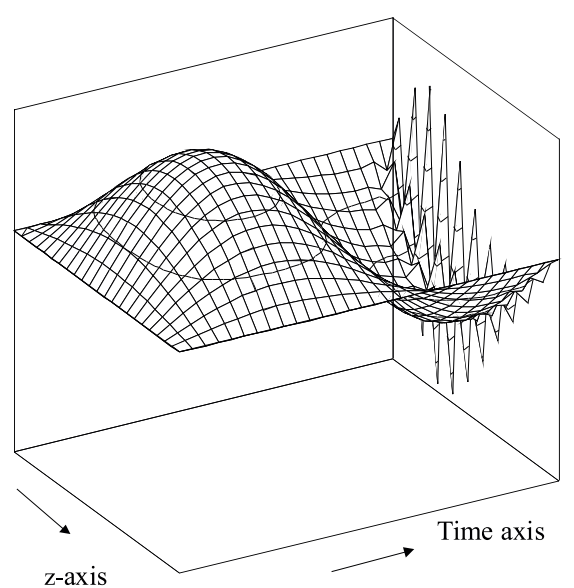

Fig. 4. Time oscillations of a half-period sinusoidal space-distribution standing wave, for $k \Delta_{z}=\pi / 22, b=c \Delta_{t} / \Delta_{z}=1.6$, $v_{p} / c=1.0013$

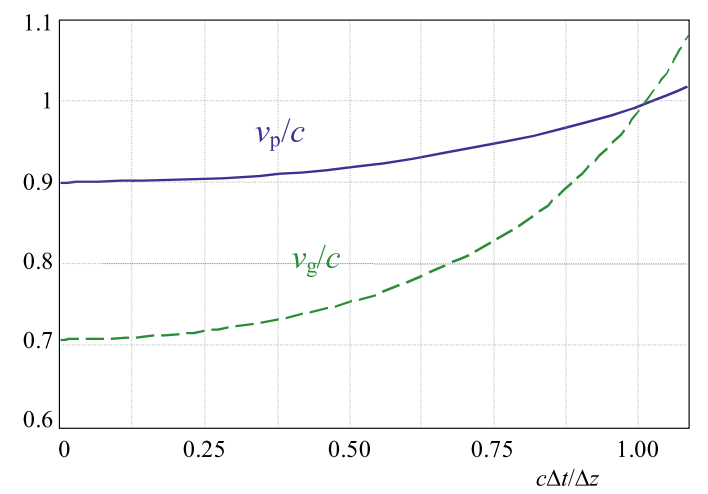

Fig. 5. The normalised phase velocity $v_{p} / c$ and the normalised group velocity $v_{g} / c$ dependence on the Courant number $b=$ $c \Delta_{t} / \Delta_{z}$ for the explicit method, $k \Delta_{z}=\pi / 2$

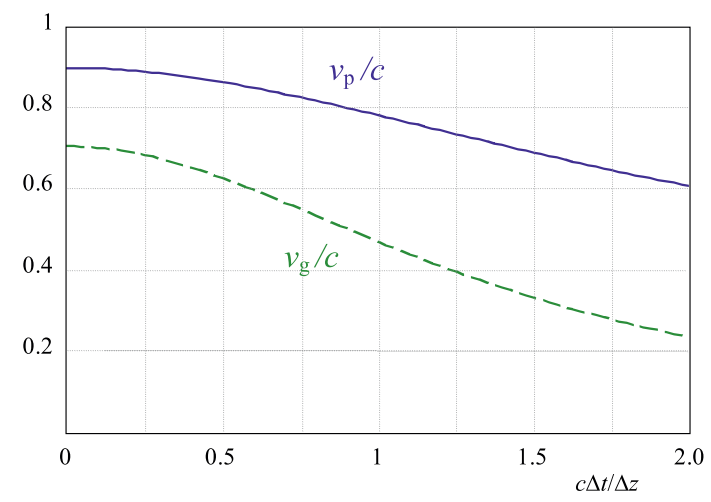

Fig. 6. The normalised phase velocity $v_{p} / c$ and the normalised group velocity $v_{g} / c$ dependence on the Courant number $b=$ $c \Delta_{t} / \Delta_{z}$ for the implicit method, $k \Delta_{z}=\pi / 2$

positive direction of the $z$-axis and having the wavenumber $k, k=2 \pi / \lambda$, where $\lambda$ is the wavelength, is given as

$$
f(z, t)=\exp (j \omega t) \exp (-j k z) .
$$

Due to (15) the "dispersion relation"

$$
\omega=c k
$$

holds, defining thus the phase velocity $v_{f}=\omega / k=c$, and the group velocity $v_{g}=\mathrm{d} \omega / \mathrm{d} k=c$ of the wave, both constant, independent of the frequency or wavenumber.

Taking the discretised expression (51), ie

$$
\left.f\right|_{m} ^{n}=\exp \left(j \omega n \Delta_{t}\right) \exp \left(-j k m \Delta_{z}\right),
$$

in the form

$$
\left.f\right|_{m} ^{n}=\xi^{n} \exp \left(-j k m \Delta_{z}\right),
$$

where $k$ is the wavenumber of single spatial harmonics, one can, using (39), investigate when the absolute value $|\xi|$ is smaller, larger or equal to one, corresponding thus to the wave power attenuation, wave power amplification, or wave power conservation (the von Neumann stability analysis). From the phase of $\xi$ one can infer also the phase and group velocity pertaining to the particular frequency $\omega$ or wavenumber $k$. This procedure called the von Neumann stability analysis was briefly described in [10] and more rigorously in [11].

Substitution of (54) into (39) yields the equation

$$
\xi^{2}-2 B \xi+1=0
$$

where

$$
B=1-2 b^{2} \sin ^{2}\left(k \Delta_{z} / 2\right),
$$

with $b=c \Delta_{t} / \Delta_{z}$ the Courant number (43). The solution of (55) reads

$$
\xi=B+j \sqrt{1-B^{2}},
$$

with $\xi$ of unit magnitude, $|\xi|=1$, only if $B^{2} \leqslant 1$, $i e-1 \leqslant B \leqslant 1$ leading thus to the condition

$$
\left(c \Delta_{t} / \Delta_{z}\right) \sin \left(k \Delta_{z} / 2\right) \leqslant 1 .
$$

In the sense of the sampling theorem the maximum wavenumber $k_{\max }$ representable by the sampling interval $\Delta_{z}$ is $k_{\max }=\pi / \Delta_{z}$. This leads to $\left.\sin \left(k \Delta_{z} / 2\right)\right|_{\max }=1$, giving thus the condition for the power conservation

$$
c \Delta_{t} \leqslant \Delta_{z}, \quad b \leqslant 1
$$

called the Courant-Friedrichs-Lewy (CFL) condition [12]. Since these highest wavenumber components are always inherently present in any shape of wave amplitude distribution - at least in form of numerical noise due to round-off errors — (59) must be considered as an ultimate upper limit.

Notice that if the zero boundary conditions are applied then the maximum wavenumber representable by the sampling interval $\Delta_{z}$ is $k_{\max }=\pi / 2 \Delta_{z}$, leading to the ultimate limit

$$
c \Delta_{t} \leqslant \sqrt{2} \Delta_{z}, \quad b \leqslant \sqrt{2} .
$$

Only if the condition of power conservation $|\xi|=1$ is met, $\xi$ can be put equal to $\xi=\exp \left(j \omega \Delta_{t}\right)$ as in (53) with

$$
\operatorname{phase}(\xi)=\omega \Delta_{t}=\arccos (B)
$$


or

$$
\cos \left(\omega \Delta_{t}\right)=1-2 b^{2} \sin ^{2}\left(k \Delta_{z} / 2\right) .
$$

We thus arrive for numerical simulation of the wave phenomena to the dispersion relation between $\omega$ and $k$

$$
\omega(k)=\frac{2}{\Delta_{t}} \arcsin \left\{b \sin \left(k \Delta_{z} / 2\right)\right\} .
$$

or written in other well-kown form [5-8]

$$
\frac{\sin \left(\omega \Delta_{t} / 2\right)}{c \Delta_{t}}=\frac{\sin \left(k \Delta_{z} / 2\right)}{\Delta_{z}} .
$$

Observe that for the limiting values $k \Delta_{z}=\pi, b=$ $c \Delta_{t} / \Delta_{z}=1$, ie for $b \sin \left(k \Delta_{z} / 2=1\right.$ in (63), the maximum representable frequency $\omega_{\max }=\pi / \Delta_{t}$, pertaining to the time step $\Delta_{t}$ in accordance with the sampling theorem, is reached.

The wavenumber-dependent phase and group velocity, $v_{p}(k)=\omega(k) / k, v_{g}(k)=\mathrm{d} \omega(k) / \mathrm{d} k$ for the numericallymodeled wave propagation becomes

$$
\begin{gathered}
v_{p}(k)=\frac{2}{k \Delta_{t}} \arcsin \left\{b \sin \left(k \Delta_{z} / 2\right)\right\} \\
v_{g}(k)=c \frac{\cos \left(k \Delta_{z} / 2\right)}{\sqrt{1-b^{2} \sin ^{2}\left(k \Delta_{z} / 2\right)}} .
\end{gathered}
$$

The above discussed effects are purely numerical artefacts, termed numerical dispersion and numerical power amplification. They can lead in case of waves consisting of more frequency components to the deterioration of the amplitude and phase distribution in the course of the wave propagation simulation.

Note that only for $b=c \Delta_{t} / \Delta_{z}=1$ the true physical values $v_{p}=c$ and $v_{g}=c$ are reached as seen from (63), (65), and (66), ie for $b=1$ the numerical simulation is free from numerical artefacts.

The effect of the power amplification is illustrated in Figure 4, where the time oscillation of a half-period sinusoidal-space-distribution standing-wave along the $z$ axis for 29 time steps with the parameter $k \Delta_{z}=\pi / 22$, and the Courant number $b=c \Delta_{t} / \Delta_{z}=1.6$, is depicted. The maximum representable wavenumber due to the sampling theorem $k_{\max }$, pertaining to the zero boundary conditions is $k_{\max } \Delta_{z}=\pi / 2$. From (56) it gives $B=-1.56$ and $|\xi| \approx 2.757$. After 29 steps this leads to the overall amplification factor of $|\xi|^{29} \approx 5.9 \times 10^{12}$. The numerical noise due to the round-off errors on approximately 14th15 th decimal place (corresponding to the 64-bit doubleprecision machine representation of real numbers), leads after the amplification to sharp random oscillations visible in Fig. 4.

The von Neumann analysis applied to implicit discretisation yields

$$
\xi^{2}-2 D \xi+1=0
$$

where

$$
D=\frac{1-b^{2} \sin ^{2}\left(k \Delta_{z} / 2\right)}{1+b^{2} \sin ^{2}\left(k \Delta_{z} / 2\right)}
$$

Observe that $D$ in (68) approaches $B$ in (56) for

$b \sin \left(k \Delta_{z} / 2\right) \ll 1$. The solution of $(67)$ yields

$$
\xi=\frac{1-b^{2} \sin ^{2}\left(k \Delta_{z} / 2\right)+2 j b \sin \left(k \Delta_{z} / 2\right)}{1+b^{2} \sin ^{2}\left(k \Delta_{z} / 2\right)}
$$

with $|\xi|=1$ independently of any condition, and

$$
\operatorname{phase}(\xi)=\omega \Delta_{t}=\arccos \frac{1-b^{2} \sin ^{2}\left(k \Delta_{z} / 2\right)}{1+b^{2} \sin ^{2}\left(k \Delta_{z} / 2\right)}
$$

leading instead of (63) to the dispersion relation of the form

$$
\frac{\tan \left(\omega \Delta_{t} / 2\right)}{c \Delta_{t}}=\frac{\sin \left(k \Delta_{z} / 2\right)}{\Delta_{z}}
$$

Now

$$
\omega(k)=\frac{2}{\Delta_{t}} \arctan \left\{b \sin \left(k \Delta_{z} / 2\right)\right\}
$$

holds, hence for the limiting value of the explicit algorithm $b \sin \left(k \Delta_{z} / 2\right)=1$ in (63) the implicit algorithm leads to $\omega \Delta_{t}=\pi / 2$ only, $i e$ for the same step $\Delta_{t}$ only to the half frequency value as compared to the explicit algorithm. The maximum representable frequency value $\omega_{\max }=\pi / \Delta_{t}$ is reached in the limit,$b=c \Delta_{t} / \Delta_{z} \rightarrow \infty$ as given by (71).

There is no limitation of the kind of CFL condition (59) upon the implicit formulation, ie the implicit method is always absolutely power conserving.

The phase and the group velocity $v_{p}$, and $v_{g}$ read

$$
\begin{gathered}
v_{p}(k)=\frac{2}{k \Delta_{t}} \arctan \left\{b \sin \left(k \Delta_{z} / 2\right)\right\}, \\
v_{g}(k)=c \frac{\cos \left(k \Delta_{z} / 2\right)}{1+b^{2} \sin ^{2}\left(k \Delta_{z} / 2\right)} .
\end{gathered}
$$

For both, explicit as well as for the implicit method, in the limit $\Delta_{t} \rightarrow 0$, the velocities $v_{p}$ and $v_{g}$ reach the values $v_{p} \approx 2 c \sin \left(k \Delta_{z} / 2\right) / k \Delta_{z}$ and $v_{g} \approx c \cos \left(k \Delta_{z} / 2\right)$. Character of the dependence of $v_{p}$ and $v_{g}$ on $\Delta_{t}$, as seen from Fig. 5 and 6 , is increasing for the explicit and decreasing for the implicit method.

\section{DISPERSION-FREE MEDIUM WITH LOSSES}

The telegraphist equations for transmission lines with losses read accordingly (1) and (2)

$$
\begin{aligned}
& L_{0} \frac{\partial i}{\partial t}+R_{0} i=-\frac{\partial u}{\partial z} \\
& C_{0} \frac{\partial u}{\partial t}+G_{0} u=-\frac{\partial i}{\partial z}
\end{aligned}
$$

With the choice of parameter-ratio $L_{0} G_{0}=R_{0} C_{0}$ representing the dispersion-free transmission line, the wave impedance remains equal to $Z_{0}=\sqrt{L_{0} / C_{0}}$, and the 
phase velocity remains equal to $c=\left(L_{0} C_{0}\right)^{-1 / 2}$. The attenuation factor will be $\alpha=\beta=\sqrt{R_{0} G_{0}}$. Such a transmission line, free of physical dispersion effects, enables one to investigate the influence of losses on the properties of discretised numerical procedures of wave propagation simulation. Moreover, in such a case the junction between lossless line and a lossy line remains reflectionfree and the wave passes completely from the lossless line into the lossy line and will be gradually attenuated there.

In the normalised form instead of (14) one obtains

$$
\begin{aligned}
& \frac{\partial f(z, t)}{c \partial t}+\alpha f(z, t)=-\frac{\partial g(z, t)}{\partial z} \\
& \frac{\partial g(z, t)}{c \partial t}+\alpha g(z, t)=-\frac{\partial f(z, t)}{\partial z}
\end{aligned}
$$

and instead of (15) the wave equation with attenuation terms

$$
\frac{\partial^{2} f(z, t)}{c^{2} \partial t^{2}}+2 \alpha \frac{\partial f(z, t)}{c \partial t}+\alpha^{2} f(z, t)-\frac{\partial^{2} f(z, t)}{\partial z^{2}}=0
$$

leading to the solution of attenuated harmonic-wave type

$$
f(z, t) \approx \exp \{j \omega(t-z / c)\} \exp (-\alpha c t)
$$

with the attenuation factor $\exp (-\alpha c t)$.

The explicitly discretised forms of (75) and (76) read

$$
\begin{aligned}
& \frac{\left.\theta_{A} f\right|_{m} ^{n+1}-\left.\theta_{S} f\right|_{m} ^{n}}{c \Delta_{t}}=-\frac{\left.g\right|_{m+1 / 2} ^{n+1 / 2}-\left.g\right|_{m-1 / 2} ^{n+1 / 2}}{\Delta_{z}} \\
& \frac{\left.\theta_{A} g\right|_{m+1 / 2} ^{n+1 / 2}-\left.\theta_{S} g\right|_{m+1 / 2} ^{n-1 / 2}}{c \Delta_{t}}=-\frac{\left.f\right|_{m+1} ^{n}-\left.f\right|_{m} ^{n}}{\Delta_{z}}
\end{aligned}
$$

where $\theta_{A, S}=1 \pm \vartheta, \vartheta=\alpha c \Delta_{t} / 2$ and the discretised wave equation (77) reads

$$
\begin{aligned}
& \frac{\left.\theta_{A}^{2} f\right|_{m} ^{n+1}-\left.2 \theta_{A} \theta_{S} f\right|_{m} ^{n}+\left.\theta_{S}^{2} f\right|_{m} ^{n-1}}{c^{2} \Delta_{t}^{2}}- \\
& \frac{f_{m+1}^{n}-\left.2 f\right|_{m} ^{n}+\left.f\right|_{m-1} ^{n}}{\Delta_{z}^{2}}=0 .
\end{aligned}
$$

The von Neumann's stability analysis yealds instead of (55) the equation

$$
(1+\vartheta)^{2} \xi^{2}-2\left(B-\vartheta^{2}\right) \xi+(1-\vartheta)^{2}=0
$$

with the solution

$$
\xi=\left\{\left(B-\vartheta^{2}\right)+j \sqrt{\left(1-\vartheta^{2}\right)^{2}-\left(B-\vartheta^{2}\right)^{2}}\right\} /(1+\vartheta)^{2},
$$

where $B$ is defined in (56) as $B=1-2 b^{2} \sin ^{2}\left(k \Delta_{z} / 2\right)$.

The value in square root in (83) must be positive, ie instead of the condition $-1 \leqslant B \leqslant 1$ leading to (58) one obtains the condition

$$
2 \vartheta^{2}-1 \leqslant B \leqslant 1
$$

leading to $b^{2} \sin ^{2}\left(k \Delta_{z} / 2\right) \leqslant 1-\vartheta^{2}$, or

$$
c \Delta_{t} \sqrt{\sin ^{2}\left(k \Delta_{z} / 2\right) / \Delta_{z}^{2}+\alpha^{2} / 4} \leqslant 1 .
$$

For $\left.\sin \left(k \Delta_{z} / 2\right)\right|_{\max }=1$ the ultimate condition reads

$$
c \Delta_{t} / \sqrt{1-\alpha^{2} c^{2} \Delta_{t}^{2} / 4} \leqslant \Delta_{z}
$$

or written alternatively $\left(c \Delta_{t}\right)^{-1} \geqslant \sqrt{1 / \Delta_{z}^{2}+\alpha^{2} / 4}$. Both (85) and (86) show that due to the attenuation terms the requirements concerning the stability of the numerical procedure are generally more stringent than condition (58).

If (85) holds, then the absolute value $|\xi|$ equals to

$$
|\xi|=|1-\vartheta| /(1+\vartheta)
$$

Since $|\xi|<1$, the attenuation of the simulated wave due to lossy transmission line always takes place. For small $\vartheta, \vartheta \ll 1$, one obtains

$$
|\xi| \approx(1-\vartheta)\left(1-\vartheta+\vartheta^{2}-\ldots\right)=1-2 \vartheta+2 \vartheta^{2}-\ldots
$$

in agreement with the first few terms of the Taylor expansion of the physical attenuation factor

$$
\exp \left(-\alpha c \Delta_{t} \approx 1-\alpha c \Delta_{t}+\left(\alpha c \Delta_{t}\right)^{2} / 2-\ldots\right.
$$

The phase term analogous to (61) is now

$$
\operatorname{phase}(\xi)=\omega \Delta_{t}=\arccos \frac{B-\vartheta^{2}}{1-\vartheta^{2}}
$$

giving for the same dimensionless parameters $k \Delta_{z}, k c \Delta_{t}$ higher value of $\omega$ in comparison with (61), ie higher phase velocity as in a lossless case.

For the implicit discretisation instead of (44) and (45) one obtains

$$
\begin{aligned}
& \frac{\left.\theta_{A} f\right|_{m} ^{n+1}-\left.\theta_{S} f\right|_{m} ^{n}}{c \Delta_{t}}= \\
& -\frac{\left.g\right|_{m+1} ^{n+1}-\left.g\right|_{m-1 / 2} ^{n+1}}{2 \Delta_{z}}-\frac{\left.g\right|_{m+1 / 2} ^{n}-\left.g\right|_{m-1 / 2} ^{n}}{2 \Delta_{z}} \\
& \frac{\left.\theta_{A} g\right|_{m+1 / 2} ^{n+1}-\left.\theta_{S} g\right|_{m+1 / 2} ^{n}}{c \Delta_{t}}= \\
& \quad-\frac{f_{m+1}^{n+1}-\left.f\right|_{m} ^{n+1}}{2 \Delta_{z}}-\frac{f_{m+1}^{n}-\left.f\right|_{m} ^{n}}{2 \Delta_{z}}
\end{aligned}
$$

and instead of (46) and (48) the set of equations

$$
\begin{aligned}
f_{m+1}^{n+1}- & \left.\left(2+4 b^{-2} \theta_{A}^{2}\right) f\right|_{m} ^{n+1}+\left.f\right|_{m-1} ^{n+1}= \\
-2\left[f_{m+1}^{n}-\left.\left(2-4 b^{-2} \theta_{A} \theta_{S}\right) f\right|_{m} ^{n}+\left.f\right|_{m-1} ^{n}\right] & -f_{m+1}^{n-1}+\left.\left(2+4 b^{-2} \theta_{S}^{2}\right) f\right|_{m} ^{n-1}-\left.f\right|_{m-1} ^{n-1}
\end{aligned}
$$



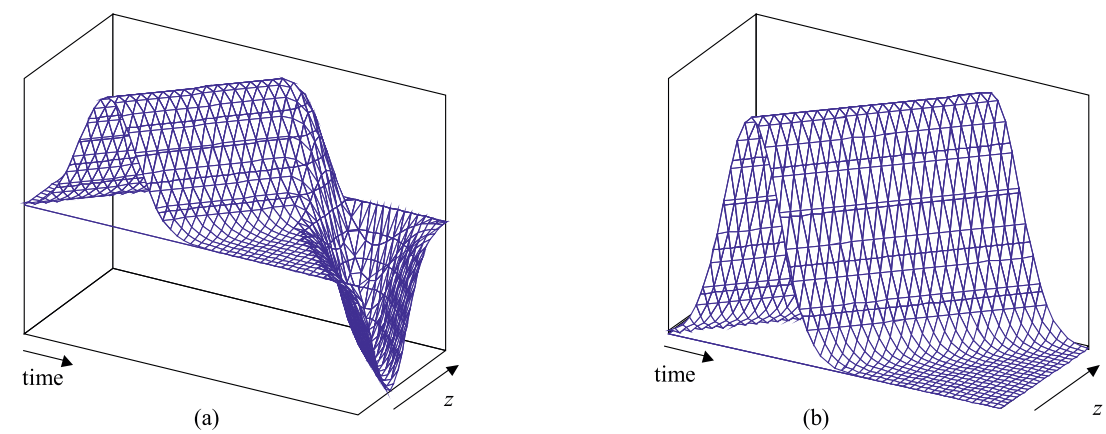

Fig. 7. Illustrative propagation of a Gaussian time-impulse excited in one boundary point to the second boundary point and its reflection (a) - on the metallic boundary (zero boundary condition), (b) - on the transparent boundary

The von Neumann's stability analysis leads now to

$$
\xi=\frac{1-b^{2} \sin ^{2}\left(k \Delta_{z} / 2\right)-\vartheta^{2}+j 2 b \sin \left(k \Delta_{z} / 2\right)}{\left\{(1+\vartheta)^{2}+b^{2} \sin ^{2}\left(k \Delta_{z} / 2\right)\right\}} .
$$

The magnitude $|\xi|$ is in contrast to the explicit method (87) given by

$$
|\xi|=\frac{\sqrt{\left[\left(1+\vartheta^{2}\right)+b^{2} \sin ^{2}\left(k \Delta_{z} / 2\right)\right]^{2}-4 \vartheta^{2}}}{(1+\vartheta)^{2}+b^{2} \sin ^{2}\left(k \Delta_{z} / 2\right)},
$$

ie the attenuation for the implicit method is generally lower than for the explicit method. The phase term $\operatorname{phase}(\xi)=\omega \Delta_{t}$ is

$$
\omega \Delta_{t}=\arccos \frac{B-\vartheta^{2}}{\sqrt{\left[\left(1+\vartheta^{2}\right)+b^{2} \sin ^{2}\left(k \Delta_{z} / 2\right)\right]^{2}-4 \vartheta^{2}}}
$$

thus leading to the lower phase velocity of the implicit algorithm as compared to the explicit one. One easily observes that for $b^{2} \ll\left(1+\vartheta^{2}\right)$ both (95) and (96) converge to the expressions for the explicit method (87) and (90).

\section{REFLECTION-FREE BOUNDARIES OF THE COMPUTATIONAL WINDOW}

The simplest boundary conditions are the Dirichlet's zero-value, or the Neumann's zero-derivative condition. For the voltage on the transmission line they represent either the short-circuited, or the open-circuited ends of the line with the voltage-reflection-coefficient equal either to minus one or to plus one.

To achieve nearly-zero reflections of the wave on the computational window boundaries and thus to mimic an infinitely long line (i.e. an infinitely large computational window) the wave must be either sufficiently attenuated when impinging on the boundaries of the computational window, or the boundary condition must be formulated in such a way as to allow the wave to virtually disappear from the computational window through its boundaries.
In the first case an absorbing layer must be placed near the boundaries in combination with the Dirichlet or Neumann's boundary condition. In the second the transparent boundary conditions have to be constructed that allow the impinging wave to out-propagate through the boundary away from the computational window without being reflected back.

The absorbing boundary condition consists in the modification of the line parameters within certain part of the line near the termination point including loss terms in order to attenuate penetrating wave.

The transparent boundaries [13] are based on the formal factorization of the wave equation (15) into two oneway wave equations, $i e$ on the formal operator product

$$
\left[\frac{1}{c} \frac{\partial}{\partial t}-\frac{\partial}{\partial z}\right]\left[\frac{1}{c} \frac{\partial}{\partial t}+\frac{\partial}{\partial z}\right] f(z, t)=0 .
$$

The outgoing wave on the "left" boundary is of the type $\exp \{j(\omega t+k z)\}$ while on the "right" boundary the outgoing wave of the type $\exp \{j(\omega t-k z)\}$. They separately fulfil the equation

$$
\left[\frac{1}{c} \frac{\partial}{\partial t}-\frac{\partial}{\partial z}\right] f(z, t)=0,
$$

on the "left" boundary, or the equation

$$
\left[\frac{1}{c} \frac{\partial}{\partial t}+\frac{\partial}{\partial z}\right] f(z, t)=0
$$

on the "right" boundary, giving thus zero value to (97).

The transparent condition on the eg "right" boundary can be implemented by either of the following formulas

$$
\begin{gathered}
\frac{\left.f\right|_{M} ^{n+1}-\left.f\right|_{M} ^{n}}{c \Delta_{t}}+\frac{\left.f\right|_{M} ^{n}-\left.f\right|_{M-1} ^{n}}{\Delta_{z}}=0 \\
\frac{\left.f\right|_{M} ^{n+1}-\left.f\right|_{M} ^{n}}{c \Delta_{t}}+\frac{\left.f\right|_{M-1} ^{n+1}-\left.f\right|_{M-1} ^{n}}{c \Delta_{t}}+ \\
\frac{\left.f\right|_{M} ^{n}-\left.f\right|_{M-1} ^{n}}{\Delta_{z}}+\frac{\left.f\right|_{M} ^{n+1}-\left.f\right|_{M-1} ^{n+1}}{\Delta_{z}}=0
\end{gathered}
$$


The first is the simple discrete explicit form of (99) using forward differences in time and backward differences in space, the second is the centered formula

$$
\frac{\left.f\right|_{M-1 / 2} ^{n+1}-\left.f\right|_{M-1 / 2} ^{n}}{c \Delta_{t}}+\frac{\left.f\right|_{M} ^{n+1 / 2}-\left.f\right|_{M-1} ^{n+1 / 2}}{\Delta_{z}}=0
$$

with approximated values $\left.f\right|_{M-1 / 2}$ by $\left(\left.f\right|_{M-1}+\left.f\right|_{M}\right) / 2$ and $\left.f\right|_{\ldots} ^{n+1 / 2}$ by $\left(\left.f\right|_{\ldots} ^{n+1}+\left.f\right|_{\ldots} ^{n}\right) / 2$.

In the case of the explicit algorithm (42) the boundary value $\left.f\right|_{M} ^{n+1}$ in the "actual" $n+1$ time layer can be determined directly from the known values in the previous time layer $\left.f\right|_{\ldots} ^{n}$ and eventually already calculated values $\left.f\right|_{\ldots} ^{n+1}$ in the "actual" time layer, ie for both (100) and (101) respectively we have

$$
\begin{gathered}
\left.f\right|_{M} ^{n+1}=\left.(1-b) f\right|_{M} ^{n}+\left.b f\right|_{M-1} ^{n}, \\
\left.f\right|_{M} ^{n+1}=\left.f\right|_{M-1} ^{n}+\frac{1-b}{1+b}\left(\left.f\right|_{M} ^{n}-\left.f\right|_{M-1} ^{n+1}\right),
\end{gathered}
$$

where $b=c \Delta_{t} / \Delta_{z}$. Note that if $b=1$ then both formulas are identical.

For the numerical implementation of implicit-type either (103), or (104) must be substituted for $\left.f\right|_{M} ^{n+1}$ in the RHS of the last equation in (49). Hence, the last equation in (49) will be for eg (104) modified to

$$
\begin{aligned}
\left.f\right|_{M-2} ^{n+1}-\left.[\eta+(1-b) /(1+b)] f\right|_{M-1} ^{n+1}= \\
\left.A\right|_{M-1} ^{n}-\left.f\right|_{M-1} ^{n}-\left.(1-b) f\right|_{M} ^{n} /(1+b) .
\end{aligned}
$$

After having solved (49) the boundary value in the "next" layer, $\left.f\right|_{M} ^{n+1}$, will be a-posteriori determined from (104).

Unfortunately, the simple formula (103) is not power conserving, since substituting (54) into (103) (the von Neumann's analysis) leads to

$$
\xi=1+j 2 b \exp \left(j k \Delta_{z} / 2\right) \sin \left(k \Delta_{z} / 2\right)
$$

with $|\xi|>1$. For (104) one obtains

$$
\xi=\frac{\cos \left(k \Delta_{z} / 2\right)+j b \sin \left(k \Delta_{z} / 2\right)}{\cos \left(k \Delta_{z} / 2\right)-j b \sin \left(k \Delta_{z} / 2\right)}
$$

ie $|\xi|=1$ and thus (104) represents an absolutely power conserving algorithm.

The quality of the transparent boundary can be judged by substituting $f(z, t) \approx \exp (j \omega t)\{\exp (-j k z)+$ $\rho \exp (j k z)\}$ into (104) and solving for the reflection coefficient $\rho$. It yields

$$
\begin{aligned}
& \rho=\exp \left(j k \Delta_{z}\right) \times \\
& \frac{b \cos \left(\frac{1}{2} \omega \Delta_{t}\right) \sin \left(\frac{1}{2} k \Delta_{z}\right)-\sin \left(\frac{1}{2} \omega \Delta_{t}\right) \cos \left(\frac{1}{2} k \Delta_{z}\right)}{b \cos \left(\frac{1}{2} \omega \Delta_{t}\right) \sin \left(\frac{1}{2} k \Delta_{z}\right)+\sin \left(\frac{1}{2} \omega \Delta_{t}\right) \cos \left(\frac{1}{2} k \Delta_{z}\right)}
\end{aligned}
$$

with $|\rho| \leqslant 1$. For $b=1$ the ideal case is achieved, there is no reflection on the boundary and $|\rho|=0$. When $b \neq 1$, and $k \Delta_{z}=\pi$ ie for the maximum representable wavenumber in accordance with the sampling theorem, the case $\rho=-1$ occurs, ie the wave is fully reflected with the opposite phase. The illustrative demonstration of the comparison between the zero boundary conditions and the transparent boundaries is in Fig. 7 .

\section{CONCLUSIONS}

In this first part of the short tutorial the selected aspects of computer simulation of electromagnetic wave phenomena in one-dimension have been thoroughly discussed. It serves the purpose as an introduction to the complex envelope FDTD formulation and to the more complicated case of electromagnetic wave propagation in two and three spatial dimensions.

\section{REFERENCES}

[1] MitCHELL, A. R. : Computational Methods in Partial Differential Equations, John Wiley \& Sons, New York, 1976.

[2] MITCHELL, A. R.-GRIFFITHS, D. F. : The Finite Difference Method in Partial Differential Equations, John Wiley \& Sons, New York, 1980

[3] STRIKWERDA, J. C.: Finite Difference Schemes and Partial Differential Equations, Wadsworth \& Brooks, Belmont, 1989.

[4] THOMAS, J. W.: Numerical Partial Differential Equations: Finite Difference Methods, Springer-Verlag, New York, 1995.

[5] TAFlOVE, A.: Computational Electromagnetics: The Finite-Difference Time-Domain Method, Artech House, Norwood, 1995.

[6] GARG, R. : Analytical and Computational Methods in Electromagnetics, Artech House, Norwood, 2008.

[7] TATSUO ITOH: Numerical Techniques for Microwave and Milimeter-Wave Passive Structures, John Wiley \& Sons, New York, 1989.

[8] BOOTON, R. C. Jr.: Computational Methods for Electromagnetics and Microwaves, John Wiley \& Sons, 1992.

[9] KANE, S. YEE : Numerical Solution of Initial boundary Value Problems Involving Maxwell's Equations in Isotropic Media, IEEE Trans. on Antennas and Prop. AP-14 No. 3 (1966), 302-307.

[10] CRANK, J.-NICOLSON, P.: A Practical Method for Numerical Evaluation of Solutions of Partial Differential Equations of Heat Conduction Type, Proc. Camb. Phil. Soc. 43 (1947), 50-67.

[11] CHARNEY, J. G.-FJORTOFT, R.-VON NEUMANN, J. : Numerical Integration of the Barotropic Vorticity Equation, Tellus 2 (1950), 273-254.

[12] COURANT, R.-FRIEDRICHS, K.-LEWY, H. : Über die partiellen Differenzengleichungen der mathematischen Physik, Math. Ann. 100 No. 1 (1928), 32-74.

13] MUR, G. : Absorbing Boundary Conditions for the Finite Difference Approximation of the Time-Domain Electromagnetic-Field Equations, IEEE Trans. on Electrmag. Compatibility EMC-23 No. 4 (1981), 377-382.

Received 6 January 2013

Lubomír Šumichrast is with the Faculty of Electrical Engineering and Information Technology of the Slovak University of Technology since 1971, now holding the position of an Associate Professor and Deputy director of the Institute of Electrical Engineering. He spent the period 1990-1992 as a visiting professor at the University Kaiserslautern, Germany and spring semester 1999 as a visiting professor at the Technical University Ilmenau, Germany. His main research interests include the electromagnetic waves propagation in various media and structures, computer modelling of wave propagation effects as well as optical communication and integrated optics. 